\title{
Electromagnetic SERS effect in carbon nanotube systems
}

\author{
I.V. Bondarev and A.V. Gulyuk \\ Math $\& 5$ Physics Department, North Carolina Central University, 1801 Fayetteville Str, \\ Durham, NC 27707, USA
}

\begin{abstract}
Quantum electrodynamics theory of the resonance Raman scattering is developed for an atom in a close proximity to a carbon nanotube. The theory describes both weak and strong atomic coupling to nanotube plasmon near fields, and predicts a dramatic enhancement of the Raman intensity in the strong coupling regime. This resonance scattering is a manifestation of the surface enhanced Raman scattering effect, and can be used in designing efficient nanotube based optical sensing substrates for single atom detection, precision spontaneous emission control, and manipulation.
\end{abstract}

Keywords: Carbon nanotubes, Interband plasmons, SERS

Surface-enhanced Raman spectroscopy (SERS) has received much of attention recently due to a very broad range of its applications ranging from optics and plasmonics to biochemistry and medicine [1, 2]. High scattering intensities within narrow spectral bands reduce the probability for spectral overlapping to allow for better recognition of multiple markers, making SERS one of the most efficient optical sensing techniques. With the development of advanced nanomaterials, various SERS substrates are demonstrated [3, 4, 5, 6, 7]. However, there is still a need for the substrates of improved sensitivity and signal reproducibility, which require clear understanding of the underlying scattering mechanisms to be developed.

\footnotetext{
${ }^{1}$ E-mail: ibondarev@nccu.edu
} 


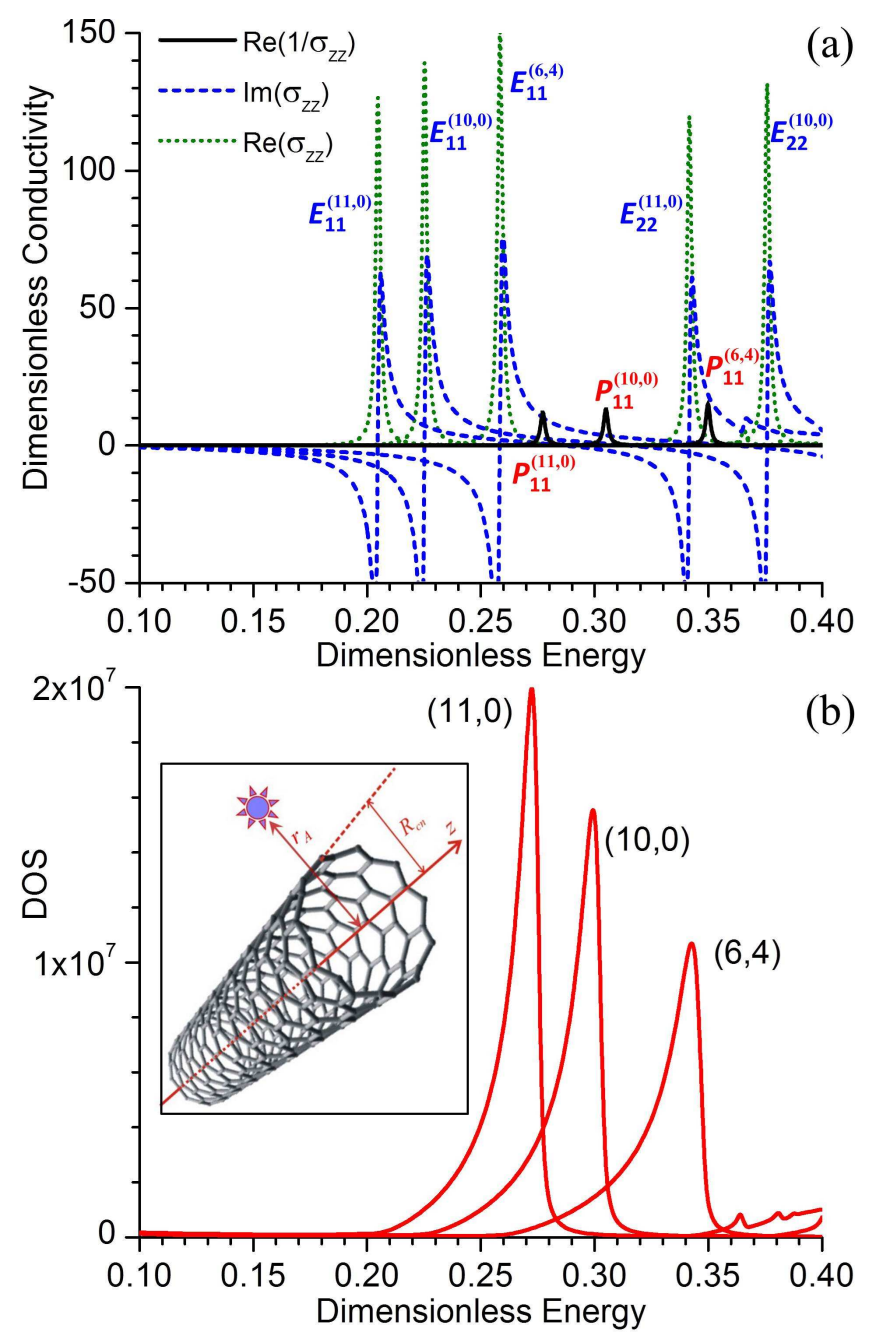

Figure 1: (Color online) (a) Fragment of the energy dependence of the dimensionless (normalized by $\left.e^{2} / 2 \pi \hbar\right)$ axial surface conductivities $\sigma_{z z}$ for the semiconducting $(6,4),(10,0)$ and $(11,0) \mathrm{CNs}$ of increasing diameter. Peaks of $\operatorname{Re} \sigma_{z z}$ represent excitons $\left(E_{11}, E_{22}, \ldots\right)$; peaks of $\operatorname{Re}\left(1 / \sigma_{z z}\right)$ are inter-band plasmons $\left(P_{11}, \ldots\right)$. (b) Photonic DOS functions for the CNs in (a) with the TLS placed at $r_{A}=R_{c n}+2 b$ from the CN symmetry axis (inset). Dimensionless energy is [Energy] $/ 2 \gamma_{0}$. Conductivities are obtained using the $(\mathbf{k} \cdot \mathbf{p})$-scheme of Ref. 9 ]. DOS functions are calculated as described in Refs. [15, 16]. 
In this article, the quantum electrodynamics (QED) theory of the resonance Raman scattering is presented for an atom near a carbon nanotube (CN). Nanotubes offer precise tunability of their electromagnetic properties by simply varying their diameters and chiralities. CNs of different diameters and chiralities feature similar electronic band structure peculiarities, yet shifted in frequency relative to one another [8, 9, 10]. This yields similar optical properties over a broad range of excitation frequencies both in the far- and in the near-field zone, originating from exciton and plasmon excitations, respectively. Excitons and plasmons are different in their physical nature, but originate from the same circumferentially quantized electronic transitions. Due to the circumferential quantization of the longitudinal electron motion, real axial (along the $\mathrm{CN}$ axis) optical conductivities of single wall CNs consist of series of peaks $E_{11}, E_{22}, \ldots$, representing the 1st, 2nd, etc. excitons, respectively [see Fig. 10(a)]. Imaginary conductivities are linked with the real ones by the Kramers-Kronig relation, and so real inverse conductivities show the resonances $P_{11}, P_{22}, \ldots$ [Fig. 1 (a)] next to their excitonic counterparts. These are interband plasmons [11, 12, 13]. Experimental evidence for such low-energy weakly-dispersive plasmon modes in CNs was first reported in Ref. 14]. These are standing charge density waves due to the periodic opposite-phase displacements of the electron shells with respect to the ion cores in the neighboring elementary cells of the CN. When excited, their associated (plasmon-induced) quasi-static fields can be strong enough to result in the enhanced Raman scattering effect by atomic type species (extrinsic atoms, ions, molecules, or semiconductor quantum dots) in the $\mathrm{CN}$ vicinity. This work derives and analyzes the differential cross-section for such scattering.

In absence of an external electromagnetic (EM) radiation, an atom, modeled here by a two-level system (TLS) positioned at the point $\mathbf{r}_{A}$ near an infinitely long single wall $\mathrm{CN}$, interacts with the quantum EM field of the $\mathrm{CN}$ via an electric dipole transition $d_{z}$ of frequency $\omega_{A}$ with the $z$-quantization axis being the CN symmetry axis [Fig. 1 (b), inset]. Transverse dipole orientations can be neglected due to the transverse depolarization effect in individual CNs. The 
Hamiltonian of the coupled CN-TLS system is given by (see Refs. [15, 16])

$$
\begin{aligned}
\hat{H} & =\hat{H}_{F}+\hat{H}_{A}+\hat{H}_{A F} \\
& =\int_{0}^{\infty} d \omega \hbar \omega \int d \mathbf{R} \hat{f}^{\dagger}(\mathbf{R}, \omega) \hat{f}(\mathbf{R}, \omega)+\frac{\hbar \tilde{\omega}_{A}}{2} \hat{\sigma}_{z} \\
& +\int_{0}^{\infty} d \omega \int d \mathbf{R}\left[\mathrm{g}^{(+)}\left(\mathbf{r}_{A}, \mathbf{R}, \omega\right) \hat{\sigma}^{\dagger}-\mathrm{g}^{(-)}\left(\mathbf{r}_{A}, \mathbf{R}, \omega\right) \hat{\sigma}\right] \hat{f}(\mathbf{R}, \omega)+\text { h.c. }
\end{aligned}
$$

with the three terms representing the (medium-assisted) quantum EM field of the CN, the TLS, and their interaction, respectively. Here, $\hat{f}^{\dagger}(\mathbf{R}, \omega)$ and $\hat{f}(\mathbf{R}, \omega)$ are the scalar bosonic field operators defined on the surface of the $\mathrm{CN}[\mathbf{R}=$ $\left(R_{c n}, \varphi, z\right)$ is the radius-vector of a point on the $\mathrm{CN}$ surface] to represent the CN field subsystem. Pauli operators, $\hat{\sigma}_{z}=|u\rangle\langle u|-| l\rangle\langle l|, \hat{\sigma}=| l\rangle\langle u|$ and $\hat{\sigma}^{\dagger}=|u\rangle\langle l|$, describe the TLS and its (dipole) transitions between the two states, upper $|u\rangle$ and lower $|l\rangle$, with the transition frequency $\omega_{A}$ modified by the diamagnetic $\hat{\mathbf{A}}^{2}$ term (vector potential) to result in the new renormalized transition frequency $\tilde{\omega}_{A}=\omega_{A}\left[1-2 /\left(\hbar \omega_{A}\right)^{2} \int_{0}^{\infty} d \omega \int d \mathbf{R}\left|\mathrm{g}^{\perp}\left(\mathbf{r}_{A}, \mathbf{R}, \omega\right)\right|^{2}\right]$. The matrix elements of the CN field interaction with the TLS are of the form $\mathrm{g}^{( \pm)}=\mathrm{g}^{\perp} \pm\left(\omega / \omega_{A}\right) \mathrm{g}^{\|}$, where $\mathrm{g}^{\perp(\|)}\left(\mathbf{r}_{A}, \mathbf{R}, \omega\right)=-i\left(4 \omega_{A} / c^{2}\right) \sqrt{\pi \hbar \omega \operatorname{Re} \sigma_{z z}(\omega)} d_{z}{ }^{\perp(\|)} G_{z z}\left(\mathbf{r}_{A}, \mathbf{R}, \omega\right)$ with ${ }^{\perp(\|)} G_{z z}$ being the $z z$-component of the transverse (longitudinal) Green tensor (with respect to the first variable) of the $\mathrm{CN}$ assisted quantum field, and $\sigma_{z z}(\omega)$ representing the CN surface axial conductivity [Fig. 10(a)]. Functions $g^{\perp(\|)}$ have the property as follows, $\int d \mathbf{R}\left|\mathrm{g}^{\perp(\|)}\left(\mathbf{r}_{A}, \mathbf{R}, \omega\right)\right|^{2}=\left(\hbar^{2} / 2 \pi\right)\left(\omega_{A} / \omega\right)^{2} \Gamma_{0}(\omega) \xi^{\perp(\|)}\left(\mathbf{r}_{A}, \omega\right)$, where $\xi^{\perp(\|)}\left(\mathbf{r}_{A}, \omega\right)=\operatorname{Im}^{\perp(\|)} G_{z z}^{\perp(\|)}\left(\mathbf{r}_{A}, \mathbf{r}_{A}, \omega\right) / \operatorname{Im} G_{z z}^{0}(\omega)$ is the transverse (longitudinal) photonic density of states (DOS) as seen from the TLS location relative to vacuum, and $\Gamma_{0}(\omega)=8 \pi \omega^{2} d_{z}^{2} \operatorname{Im} G_{z z}^{0}(\omega) / \hbar c^{2}$ is the TLS spontaneous decay rate in vacuum with $\operatorname{Im} G_{z z}^{0}(\omega)=\omega / 6 \pi c$ being the vacuum imaginary Green tensor $z z$-component.

Hamiltonian (11) involves only two standard approximations, the electric dipole and two-level approximation [15, 16], while conveniently representing the coupled TLS-CN system in terms of the relative distance dependent DOS functions $\xi^{\perp(\|)}\left(\mathbf{r}_{A}, \omega\right)$. For short TLS-CN separation distances EM retardation effects play no role, and so one has $\xi^{\perp}=\xi^{\|}=\xi\left(\mathbf{r}_{A}, \omega\right)$ for the DOS func- 
tions [16]. Figure 1(b) shows the calculations of $\xi\left(r_{A}=R_{c n}+2 b, x\right), x=\hbar \omega / 2 \gamma_{0}$ being the dimensionless energy, with $b=1.42 \AA$ and $\gamma_{0}=2.7 \mathrm{eV}$ representing the C-C interatomic distance and overlap integral, respectively, for the three semiconducting CNs of increasing diameter. We see the sharp single-peak resonances originating from the interband plasmons of the respective CNs [cf. Fig.11(a) and Fig. 1(b)]. These are responsible for the CN-TLS coupling in the near-field. In the linear coupling regime, quite generally, the coupled CN-TLS system can be represented as a four-level system with the eigenvectors of the Hamiltonian (1) being as follows

$$
\begin{aligned}
|0\rangle & =|l\rangle|\{0\}\rangle, \\
|1\rangle & =C_{u}^{(1)}|u\rangle|\{0\}\rangle+\int_{0}^{\infty} d \omega \int d \mathbf{R} C_{l}^{(1)}(\mathbf{R}, \omega)|l\rangle|\{1(\mathbf{R}, \omega)\}\rangle, \\
|2\rangle & =C_{u}^{(2)}|u\rangle|\{0\}\rangle+\int_{0}^{\infty} d \omega \int d \mathbf{R} C_{l}^{(2)}(\mathbf{R}, \omega)|l\rangle|\{1(\mathbf{R}, \omega)\}\rangle, \\
|3\rangle & =|u\rangle|\{1(\mathbf{R}, \omega)\}\rangle .
\end{aligned}
$$

Here, $|\{0\}\rangle$ and $|\{1(\mathbf{R}, \omega)\}\rangle$ are, respectively, the vacuum and single-quantum excited states of the $\mathrm{CN}$ field subsystem, and $C_{u, l}^{(1),(2)}$ are the mixing coefficients of the spontaneous decay type transition $|u\rangle|\{0\}\rangle \rightarrow|l\rangle|\{1(\mathbf{R}, \omega)\}\rangle$ in the coupled CN-TLS system [17, 16], while the similar mixing of the $|l\rangle|\{0\}\rangle$ and $|u\rangle|\{1(\mathbf{R}, \omega)\}\rangle$ states, known to be responsible for the long-range dispersive van der Waals interaction [15, 16], is neglected for simplicity.

To proceed with the Raman scattering cross-section calculations, it is necessary to fully determine scatterer's eigenvectors in Eq. (2) together with their respective energy eigenvalues. Approximating the sharp DOS resonance [Fig.1(b)] at the plasmon frequency $\omega_{p}$ by the Lorentzian function of half-width-at-halfmaximum $\Delta \omega_{0}$ of the form $\xi\left(r_{A}, \omega\right) \approx \xi\left(r_{A}, \omega_{p}\right) \Delta \omega_{0}^{2} /\left[\left(\omega-\omega_{p}\right)^{2}+\Delta \omega_{0}^{2}\right]$, allows one to obtain the analytical solution to the eigenvalue problem that is valid both in the weak and in the strong CN-TLS coupling regime [18]. Namely, applying the Hamiltonian (1) to the four-vector set (2), one obtains the (dimensionless) eigen energies as follows

$$
\varepsilon_{0}=-\frac{\tilde{x}_{A}}{2}
$$




$$
\begin{aligned}
\varepsilon_{1,2} & =\frac{1}{2}\left(x_{p} \mp \sqrt{\delta^{2}+X^{2}}-i \Delta x_{p}\right) \\
\varepsilon_{3} & =\frac{\tilde{x}_{A}}{2}+x_{p}-i \Delta x_{p}
\end{aligned}
$$

where $\left(\tilde{x}_{A}, x_{p}, \Delta x_{p}\right)=\hbar\left(\tilde{\omega}_{A}, \omega_{p}, \Delta \omega_{p}\right) / 2 \gamma_{0}$ with $\Delta x_{p}$ added to phenomenologically account for the finite half-width of the plasmon resonance, as seen in Fig. 1 (a), which is assumed to be much broader than the excited atomic level natural half-width dropped here on this account for simplicity, $\delta=\tilde{x}_{A}-x_{p}$, and $X=\left(\hbar / 2 \gamma_{0}\right) \sqrt{2 \Delta \omega_{0} \Gamma_{0}\left(\omega_{p}\right)\left(1+\omega_{A}^{2} / \omega_{p}^{2}\right) \xi\left(r_{A}, \omega_{p}\right)}$. For the mixing coefficients, one has

$$
\left|C_{u}^{(1),(2)}\right|^{2}=\frac{1}{2}\left(1+\frac{1 \mp \sqrt{1+X^{2} / \delta^{2}}}{1+X^{2} / \delta^{2} \mp \sqrt{1+X^{2} / \delta^{2}}}\right)
$$

and

$$
\int_{0}^{\infty} d \omega \int d \mathbf{R}\left|C_{l}^{(1),(2)}(\mathbf{R}, \omega)\right|^{2}=\frac{\left|C_{u}^{(1),(2)}\right|^{2} X^{2} / 2 \delta^{2}}{1+X^{2} / 2 \delta^{2} \mp \sqrt{1+X^{2} / \delta^{2}}}
$$

accordingly. These equations are valid both in resonance, where $\delta \sim 0$ and so $X^{2} / \delta^{2} \gg 1$, and out of resonance where $X^{2} / \delta^{2} \ll 1$, and give different easily derivable asymptotical expressions in these two regimes of relevance to strong and weak CN-TLS coupling, respectively.

Under the assumption that the coupled CN-TLS system with the eigen states (2)-(5) is initially in the ground state, the inelastic scattering of an external EM radiation by this four-level system only involves transitions between levels $|0\rangle,|1\rangle$ and $|2\rangle$, as shown in Fig. 2, top, due to the dipole moment selection rule restrictions. The entire scattering process includes three sequential steps [19, 20]. They are: (a) excitation of the system by an incident photon of the frequency $\omega_{i}$ with the unit polarization vector $\mathbf{e}_{i}$, described by the interaction matrix element (normalized at one photon per unit volume [19]) $\left\langle n\left|\hat{H}_{R}\left(\omega_{i}\right)\right| 0\right\rangle=(-i / c) \sqrt{2 \pi \hbar \omega_{i}} d_{z} \cos \vartheta_{i} C_{u}^{(n) *}(n=1,2)$ with $\cos \vartheta_{i}=\mathbf{e}_{i} \cdot \mathbf{e}_{z} ;$ (b) plasmon emission (or absorption) on the CN surface, described by the matrix element $\left\langle 1\left|\hat{H}_{A F}^{(e)}\right| 2\right\rangle$ (or $\left.\left\langle 2\left|\hat{H}_{A F}^{(a)}\right| 1\right\rangle\right)$ with $\hat{H}_{A F}^{(e)}$ and $\hat{H}_{A F}^{(a)}$ being the emission term $\left(\sim \hat{f}^{\dagger}\right)$ and the absorption term $(\sim \hat{f})$, respectively, of the interaction Hamiltonian $\hat{H}_{A F}$ in Eq. (1); (c) de-excitation of the CN-TLS system by the scattered (Raman) photon emission of the frequency $\omega_{s}$ with the unit polariza- 


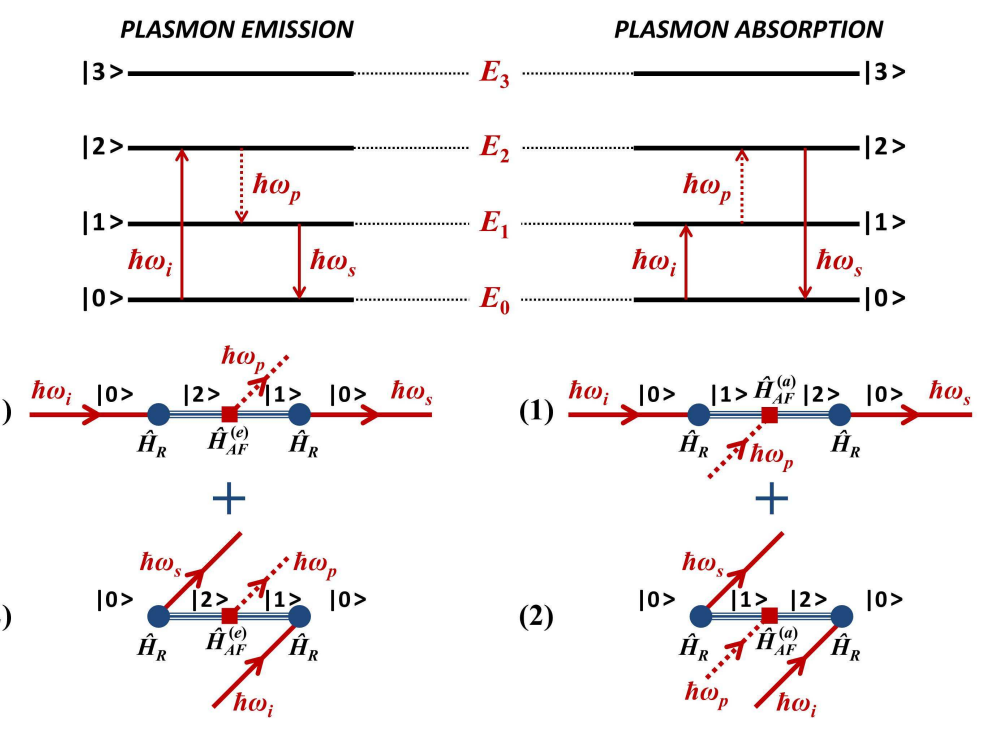

Figure 2: (Color online) Schematic of the Raman scattering process (top) in terms of the inter-level transitions of the coupled CN-TLS system given by Eqs. (2)-(5), and Feynman diagrams (bottom) for the Raman cross-section calculations.

tion vector $\mathbf{e}_{s}$, described by the interaction matrix element $\left.\left\langle n\left|\hat{H}_{R}\left(\omega_{i}\right)\right| 0\right\rangle^{*}\right|_{i \rightarrow s}$. There are four Feynman diagrams, shown in Fig. 2. bottom, to contribute to this process. They are two for the plasmon emission (bottom left) and two for the plasmon absorption (bottom right), to represent two indistinguishable ways for the emission and absorption to occur. Two types of the emission (absorption) diagrams should be summed up and squared, followed by adding the emission and absorption contributions together [19, 20]. This results in the differential Raman scattering cross-section of the form

$$
\frac{d \sigma}{d \Omega_{s}}=\frac{\left(2 \gamma_{0}\right)^{2}\left|d_{z}\right|^{4}}{\hbar^{4} c^{4}} \cos ^{2} \vartheta_{i} \cos ^{2} \vartheta_{s} P\left(x_{i}, x_{s}\right)
$$

with the dimensionless (angle-free) scattering probability

$$
\begin{gathered}
P\left(x_{i}, x_{s}\right)=\frac{x_{i} x_{s}^{3} X^{8}}{2^{6}\left(\delta^{2}+X^{2}\right)^{2}\left(\delta_{-}^{2}+\Delta x_{p}^{2}\right)} \\
\times\left\{\frac{1}{\left[\left(x_{i}-x_{p}-\delta_{+} / 2\right)^{2}+\Delta x_{p}^{2}\right]\left[\left(x_{s}-x_{p}-\delta_{-} / 2\right)^{2}+\Delta x_{p}^{2}\right]}\right.
\end{gathered}
$$




$$
\left.+\frac{1}{\left[\left(x_{i}-x_{p}-\delta_{-} / 2\right)^{2}+\Delta x_{p}^{2}\right]\left[\left(x_{s}-x_{p}-\delta_{+} / 2\right)^{2}+\Delta x_{p}^{2}\right]}\right\}
$$

where $x_{i, s}=\hbar \omega_{i, s} / 2 \gamma_{0}, \delta_{ \pm}=\delta \pm \sqrt{\delta^{2}+X^{2}}$, and only the resonant terms are left to represent the contributions from plasmon emission and absorption, respectively, while (insignificant, quasi-constant background) non-resonant terms are dropped for brevity.

Each term in Eq. (77) has the product of two resonance energy denominators that include $x_{i}$ and $x_{s}$, incident (incoming) and scattered (outgoing) photon energies. This is what makes this Raman scattering resonant. For fixed $x_{i}$, only one term contributes, resulting in either Stokes scattering with $x_{s}<x_{i}$ and a plasmon created in the $\mathrm{CN}$, or in anti-Stokes scattering with $x_{s}>x_{i}$ and a plasmon absorbed from the CN. The absolute value of the Raman shift is $\sqrt{\delta^{2}+X^{2}}$, yielding a quantity $\sim X$ in resonance, where $\delta \sim 0$ whereby $X^{2} / \delta^{2} \gg$ 1 , and that $\sim \delta$ out of resonance with $X^{2} / \delta^{2} \ll 1$. In the latter case, the prefactor in Eq. (7) tends to zero as $X^{4} / \delta^{4}$, totally ruling out the probability of the scattering process. When in resonance, on the other hand, the maximum of $P\left(x_{i}, x_{s}\right)$ goes as $\left(X^{4} / \Delta x_{p}^{4}\right) /\left(X^{2}+\Delta x_{p}^{2}\right)$, being strongly suppressed in the weak TLS-plasmon coupling regime where $X^{2} / \Delta x_{p}^{2} \ll 1$, and being dramatically enhanced in the opposite case where $X^{2} / \Delta x_{p}^{2} \gg 1$ so that the TLS-plasmon coupling is strong. The scattering enhancement factor is about square of that for the resonance absorption by atomically doped CNs [21], the way it should be for scattering as a two-step process of absorption followed by emission viewed as "reversed absorption".

Figure 3 shows an example of the numerical calculations for the scattering probability $P\left(x_{i}, x_{s}\right)$ as given by Eq. (7) for $x_{p}=0.35\left[P_{11}^{(6,4)}\right.$ plasmon in Fig. 1 (a)] with $\Delta x_{p}$ and $X$ varying independently [columns (b) and (a)] within a typical (conservative) range of parameters [21], to see the plasmon decoherence effect and the role of DOS resonance variation as the TLS-CN separation distance changes. As discussed above, Raman scattering is seen to be very sensitive to the strong TLS-plasmon coupling, blowing up by a factor over $10^{3}$ for $X / \Delta x_{p} \sim 10$ and totally vanishing when $X / \Delta x_{p} \sim 1$. Raising $X$ increase both 

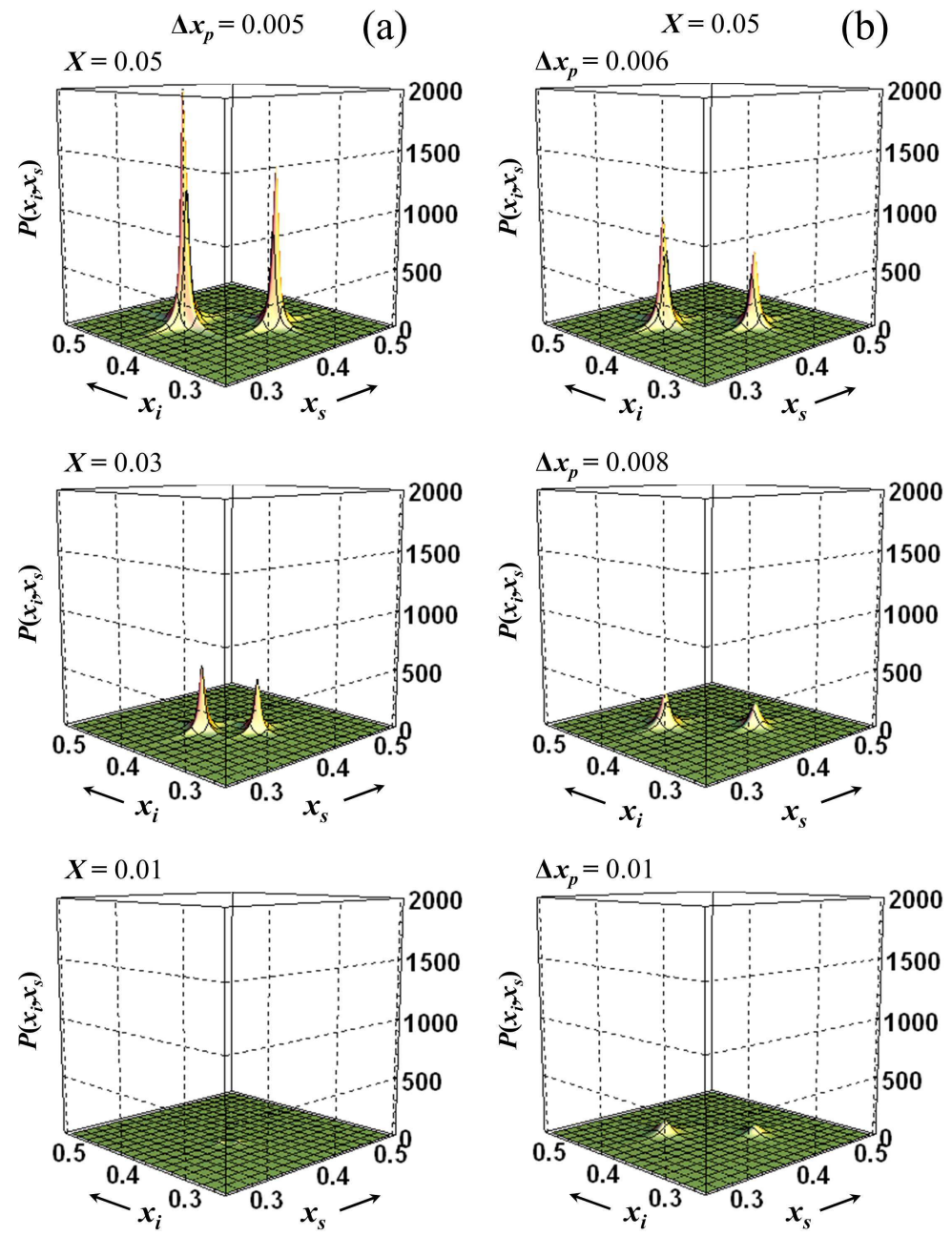

Figure 3: (Color online) Dimensionless scattering probability given by Eq. (7) for $x_{p}=0.35$ $\left[P_{11}^{(6,4)}\right.$ plasmon in Fig. 1 (a)] with $X$ and $\Delta x_{p}$ varying independently [columns (a) and (b)]. TLS-plasmon coupling strength is represented by the ratio $X / \Delta x_{p}$ being greater or less than unity for strong and weak coupling, respectively. Raman scattering effect is seen to be manifestly indicative of the strong TLS-plasmon coupling, dramatically increasing as the ratio $X / \Delta x_{p}$ goes much greater than unity and disappearing when it is comparable with unity. 
the Raman shift and the intensity, while greater $\Delta x_{p}$ quench the intensity with no Raman shift change.

In summary, the QED theory of the resonance Raman scattering is developed for a two-level dipole emitter coupled to a weakly-dispersive interband plasmon resonance of a carbon nanotube. Such scattering is a manifestation of the general SERS effect, in which the enhancement is due to plasmon-induced near-fields that affect the TLS in close proximity to the CN surface. Raman cross-section obtained covers both weak and strong TLS-plasmon coupling, and shows a dramatic increase in the strong coupling regime. The effect may be used to detect individual atomic type objects trapped near CNs. More advanced applications, which require further theoretical development, may include highly efficient CN based SERS substrates for single molecule/atom/ion detection, precision spontaneous emission control, and optical manipulation.

I.V.B. acknowledges the US Department of Energy award (DE-SC0007117). A.V.G. is supported by the US National Science Foundation (ECCS-1306871).

\section{References}

[1] K.Kneipp, M.Moscovits, and H.Kneipp, Surface-enhanced Raman scattering: Physics and applications (Springer-Verlag, Berlin, 2006).

[2] R.Zhang, Y.Zhang, Z.C.Dong, S.Jiang, C.Zhang, L.G.Chen, L.Zhang, Y.Liao, J.Aizpurua, Y.Luo, J.L.Yang, and J.G.Hou, Nature 498, 82 (2013).

[3] M.Peng, H.Xu, and M.Shao, Appl. Phys. Lett. 104, 193103 (2014)

[4] D.M.Andrada, H.S.Vieira, M.M.Oliveira, A.P.Santos, L.Yin, R.Saito, M.A.Pimenta, C.Fantini, and C.A.Furtado, Carbon 56, 235 (2013).

[5] Q.Hao, S.M.Morton, B.Wang, Y.Zhao, L.Jensen, and T.J.Huang, Appl. Phys. Lett. 102, 011102 (2013). 
[6] R.Lv, Q.Li, A.R.Botello-Mendez, T.Hayashi, B.Wang, A.Berkdemir, Q.Hao, A.L.Elias, R.Cruz-Silva, H.R Gutierrez, Y.A.Kim, H.Muramatsu, J.Zhu, M.Endo, H.Terrones, J.-C.Charlie, M.Pan, and M.Terrones, Scientific Reports 2, 586 (2012).

[7] D.Z.Lin, Y.P.Chen, P.J.Jhuang, J.Y.Chu, J.T.Yeh, and J.-K.Wang, Optics Express 19, 4337 (2011).

[8] R.Saito, G.Dresselhaus, and M.S.Dresselhaus, Science of Fullerens and Carbon Nanotubes (Imperial College Press, London, 1998).

[9] T.Ando, J. Phys. Soc. Jpn. 74, 777 (2005).

[10] T.Hertel and I.V.Bondarev (eds.), Photophysics of Carbon Nanotubes and Nanotube Composites (Special Issue), Chem. Phys. 413, 1 (2013).

[11] I.V.Bondarev, L.M.Woods, and K.Tatur, Phys. Rev. B 80, 085407 (2009).

[12] I.V.Bondarev, Phys. Rev. B 85, 035448 (2012).

[13] I.V.Bondarev and T.Antonijevic, Phys. Stat. Sol. C 9, 1259 (2012).

[14] T.Pichler, M.Knupfer, M.S.Golden, J.Fink, A.Rinzler, and R.E.Smalley, Phys. Rev. Lett. 80, 4729 (1998).

[15] I.V.Bondarev and Ph.Lambin, Phys. Rev. B 72, 035451 (2005).

[16] I.V.Bondarev and Ph.Lambin, Near-field electrodynamics of atomically doped carbon nanotubes, in: Trends in Nanotubes Reasearch (Nova Science, New York, 2006). Chapter 6, p. 139.

[17] I.V.Bondarev and Ph.Lambin, Phys. Rev. B 70, 035407 (2004).

[18] Actual DOS resonance frequencies are slightly red shifted relative to their respective plasmon resonance frequencies [cf. Figs. [1 (a) and (b)]. The shifts are within plasmon resonance widths though, and so are neglected, thereby reducing the number of relevant theory parameters here. 
[19] V.B.Berestetskii, E.M.Lifshitz, and L.P.Pitaevskii, Quantum Electrodynamics (Pergamon, Oxford, 1982).

[20] P.Y.Yu and M.Cardona, Fundamentals of Semiconductors, 4th edn. (Springer-Verlag, Berlin 2010).

[21] I.V.Bondarev and B.Vlahovic, Phys. Rev. B 74, 073401 (2006). 